\title{
The 3D Kaleidoscope
}

James F. Blinn, Caltech

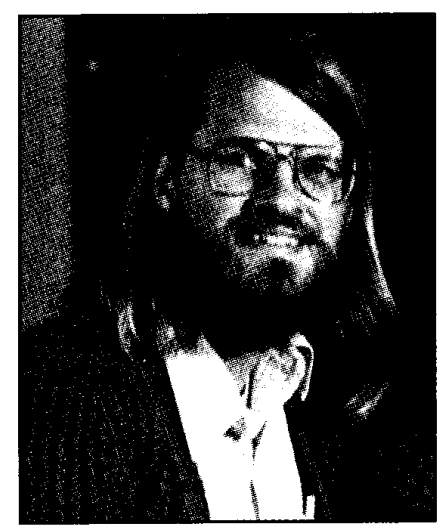

A few months ago I did a column on Platonic solids, talking mostly about the coordinates of the vertices. This month, let's examine another way to construct Platonic solids which takes advantage of their symmetry. This works well with the coordinate transform system we have developed, since symmetry is based on coordinate transformations.

There are two ways to think of symmetry: First, there is the analytic approach. You start with some symmetrical shape and characterize its symmetries as the collection of transformations it can undergo that leave its shape unchanged. The allowable transformations we will consider here are pure rotations and mirror reflections. Second is the synthetic approach: You start with some asymmetrical seed shape, and you apply the above collection of transformations to generate the whole symmetrical shape.

\section{The cube}

Let's begin with an analysis of the symmetries of a cube. A cube can be rotated by $90^{\circ}$ about, say, the $x$ axis, and the result looks identical to the original cube. Likewise it can be mirrored along the $\mathrm{x}$ axis without changing its shape. With a little organization and imagination you can discover that there are 48 different transformations that result in a cube indistinguishable from the original. These include 24 pure rotations, to wit:

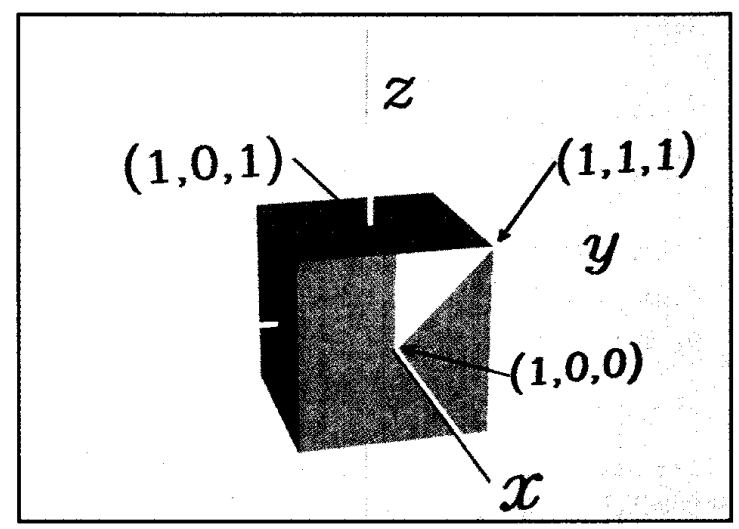

Figure 1.

- The identity

- Rotation of $90^{\circ}, 180^{\circ}$, and $270^{\circ}$ about the $x, y$, and $z$ axes (9 total)

- Rotation of $120^{\circ}$ and $240^{\circ}$ about the four diagonals (8 total)

- Rotation of $180^{\circ}$ about the axes connecting the center with the midpoints of each edge (6 total)

The other 24 transformations are reflections or rotation/reflections.

- The point inversion (scale by -1 along each of $x, y$, and $z$ axes)

- Reflection along the $x, y$, or $z$ axis, followed by rotation of $0^{\circ}, 90^{\circ}$, or $-90^{\circ}$ about that axis (9 total)

- Reflection along the four diagonals followed by rotation of $\pm 60^{\circ}$ about that diagonal (8 total)

- Reflection through the planes $x= \pm y, x= \pm z$, and $y= \pm z$ (6 total)

Next let's switch to the synthetic approach. What is the minimal asymmetrical portion of a cube that can be transformed by all these rotations and reflections to regenerate the whole cube? One choice is the triangle, shown in yellow in Figure 1, having the vertex coordinates: 


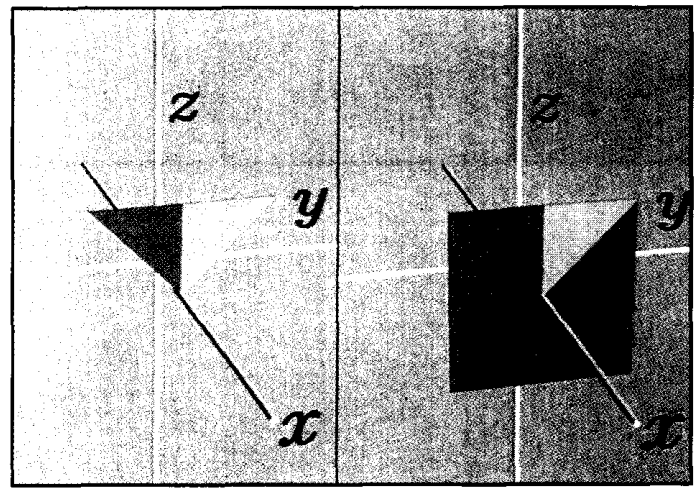

a

Figure 2.

Point 1: $(1,1,1)$

Point 2: $(1,0,0)$

Point 3: $(1,0,1)$

Now how do we build up a cube from this seed shape? It is possible (but inconvenient) to apply all 48 transformations to the triangle individually. Instead let's build it up in modules. Here's one of the many ways to do it. First combine the triangle with its mirror image in the $x y$ plane to get the symmetrical triangle in Figure $2 \mathrm{a}$. This can be described in our transformation system (from the October 1987 column) as

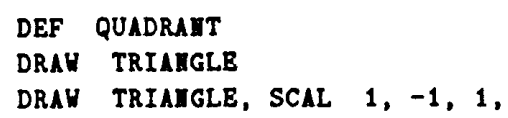

Then replicate this four times by $90^{\circ}$ rotations about the $x$ axis to get one entire face of the cube. This is shown in Figure $2 b$ and defines CFACE:

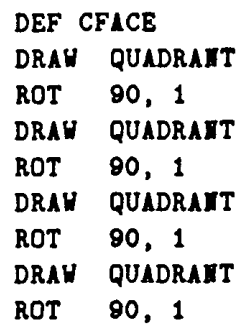

Finally generate all six sides of the cube by $90^{\circ}$ rotations about the $y$ and $z$ axes:

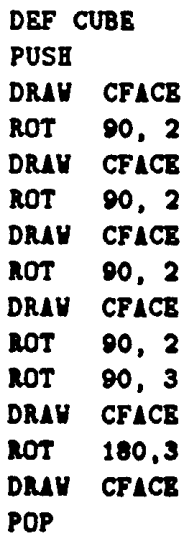

What have we just done? The collection of 48 transformations of the cube makes up a mathematical entity known as a group. One property of such a group is that it is closed under matrix multiplication, that is, after performing any two transformations in sequence, the net result is always another transformation in the group. You can then make a $48 \times 48$ multiplication table and all products will be one of the original 48. Alternatively, you can often start with just a few seed transformations and generate all the rest with multiple products of the seeds. For example, you can generate all the pure rotations of the cube group using just two matrices, $90^{\circ}$ rotation about $\mathrm{x}$ (call this $\mathrm{A}$ ) and $90^{\circ}$ about $y$ (call this $B$ ). I entertained myself for an entire weekend working out the combinations of $A$ and $B$ necessary for each of the other 22 transformations. It takes five, for example, to construct a $90^{\circ}$ rotation about $z$ (ABAAA). I also found some surprising identities ( $\mathrm{ABA}=\mathrm{BAB} ; \mathrm{AA}=\mathrm{BAAB}$; etc.). You could even express these as rewriting rules in some kind of context-free grammar

But I digress.

\section{Other related shapes}

Now that we have a way of generating the cube from transformations of our seed shape, what happens if we change the seed shape? For the time being, let's keep the shape such that its edges continue to match with the edges of its neighbors. That is, we will not introduce tears or gaps in the final shape. To do this, point 1 must remain on the xyz diagonal, point 2 must remain on the $x$ axis, and point 3 must remain on the $x z$ plane. The point coordinates can be parameterized as

Point 1: $(a, a, a)$

Point 2: $(b, 0,0)$

Point 3: $(c, 0, c)$

My implementation of the transformation system doesn't allow changing shapes in this way. Instead I did it by starting with a slightly different seed shape called BTRIANG, expressed in point-polygon form as 
PIT 1, 1.,0., 0 .

PIT 2, 0.,1.,0.

PET 3,0.,0.,1.

POLY $1,2,3$

then subjecting it to the transformation

$$
\left[\begin{array}{lll}
a & a & a \\
b & 0 & 0 \\
c & 0 & c
\end{array}\right]
$$

This appears in transformation language as

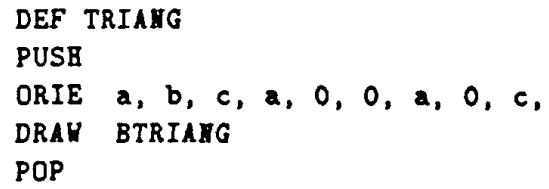

If $a=b=c=1$, we get our cube back (see Figure 3a). By playing with $a, b$, and $c$, you can get a variety of closed shapes, all sharing the same symmetry properties as the cube. What sort of other shapes can we get? One interesting shape comes from $a=\sqrt{2}, b=\sqrt{6}, c=\sqrt{3}$. Here all points are equidistant from the origin, and the object is roughly spherical (see Figure 3b). Other shapes include the Octahedron (the dual shape to the cube) (see Figure 3c), and the rhombic dodecahedron, a shape with 12 faces, each of which is a rhombus (see Figure 3d).

How can we organize this collection? One interesting way is to observe the values of $a, b$, and $c$ that cause the seed triangle to be coplanar with its three neighbors. In Figure 4 the three neighbors are colored red, green, and blue. What restrictions on the values of $a, b, c$ does coplanarity imply? The three points $(a, a, a),(b, 0,0)$, and $(c, 0, c)$ of the yellow triangle lie in the plane whose column vector is

$$
\left[\begin{array}{c}
a c \\
b c-a b \\
a b-a c \\
-a b c
\end{array}\right]
$$

The three conditions of coplanarity translate into

1. Red triangle is coplanar with yellow triangle. The point $(a,-a, a)$ lies on the plane. Dotting it with the plane vector and setting the result to zero ultimately shows that $a=c$.

2. Blue triangle is coplanar with yellow. The point $(0,0, b)$ is on the plane, which implies $2 c=b$.

3. Green triangle is coplanar with yellow. The point $(c, c, 0)$ is on the plane, which implies $a c+b c=2 a b$
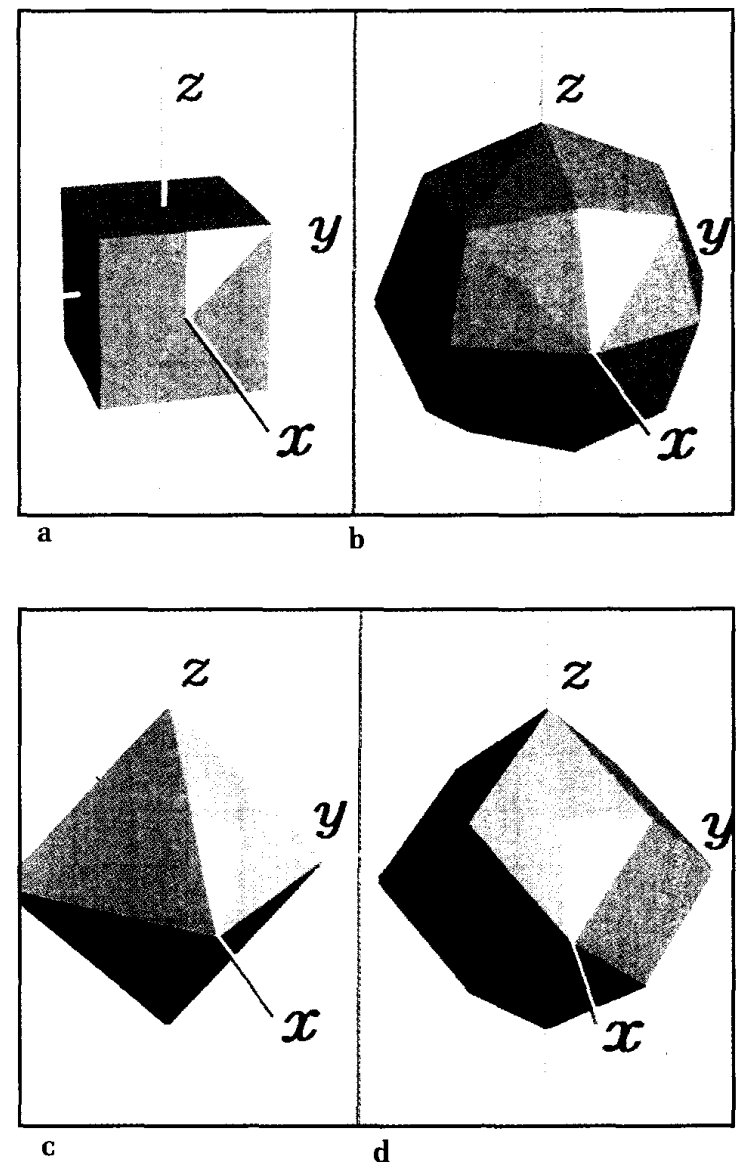

Figure 3.

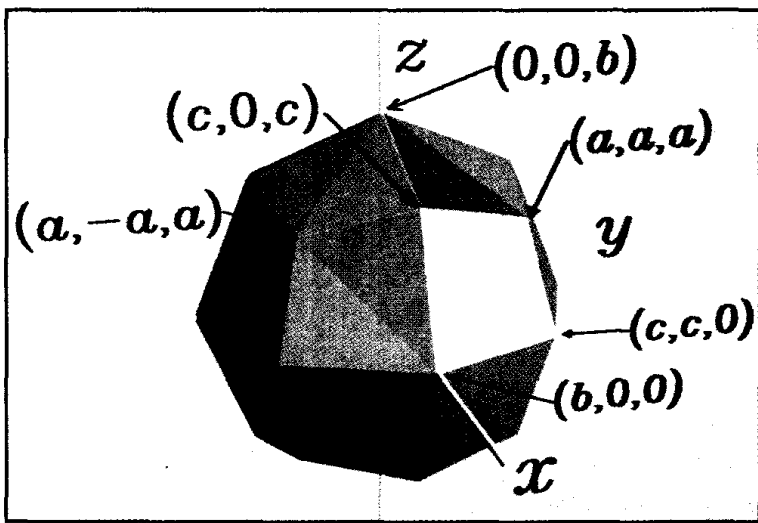

Figure 4.

We can visualize these conditions as curves in a "shape space" with axes $a, b$, and $c$. Since any scalar multiple of $a, b, c$ generates the same shape (just larger or smaller), these numbers form a sort of homogeneous coordinate system, so we will simplify the situation by fixing $c$ at the value 1 . The three conditions translate 


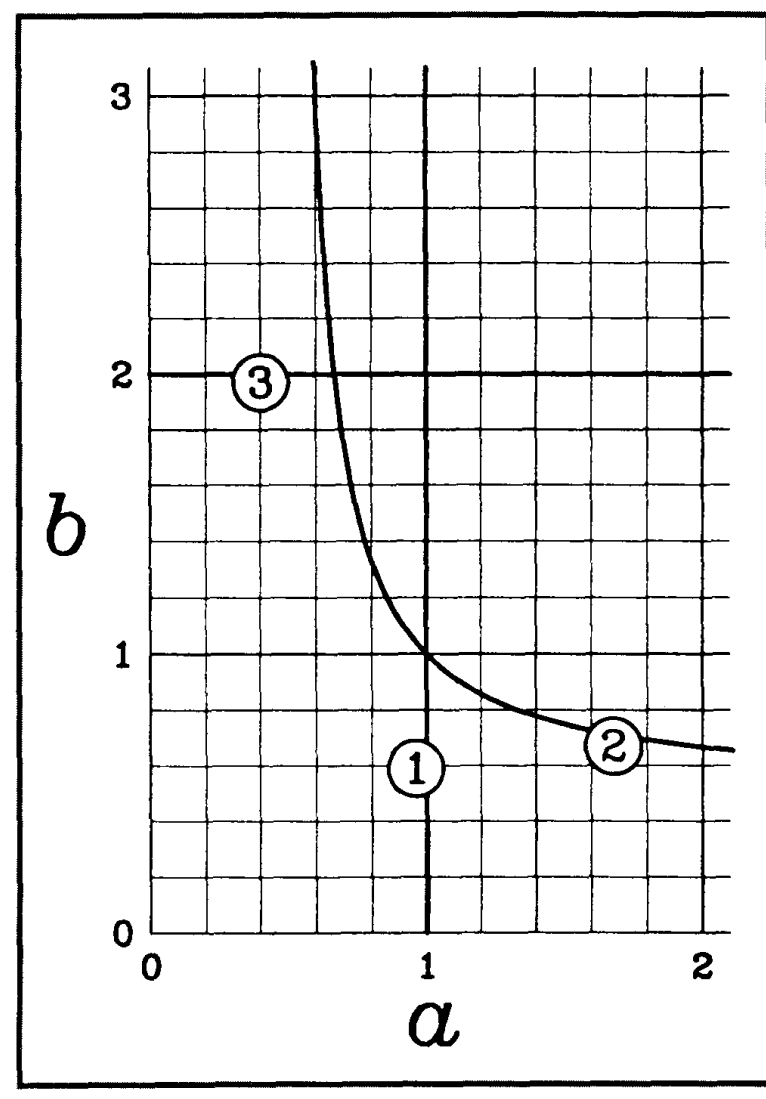

Figure 5.

into three lines in the a,b plane (see Figure 5). The conditions are
1. $a=1$
2. $b=2$
3. $b=\frac{a}{2 a-1}$

Where line 1 and 2 are met you get the cube. Where line 2 and 3 meet, you get the octahedron. Where lines 3 and 1 meet, you get the rhombic dodecahedron. Tabulating these gives

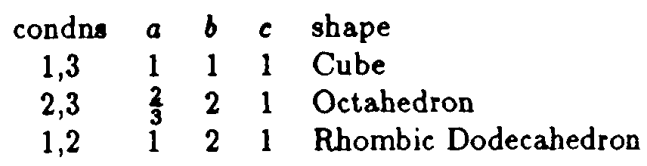

Let's play with this a bit. If you slowly interpolate a and $b$ from their cube values to their octahedron values, keeping condition 3 satisfied, you get a transformation of the cube shape to the octahedron shape, which looks particularly interesting. Since the green

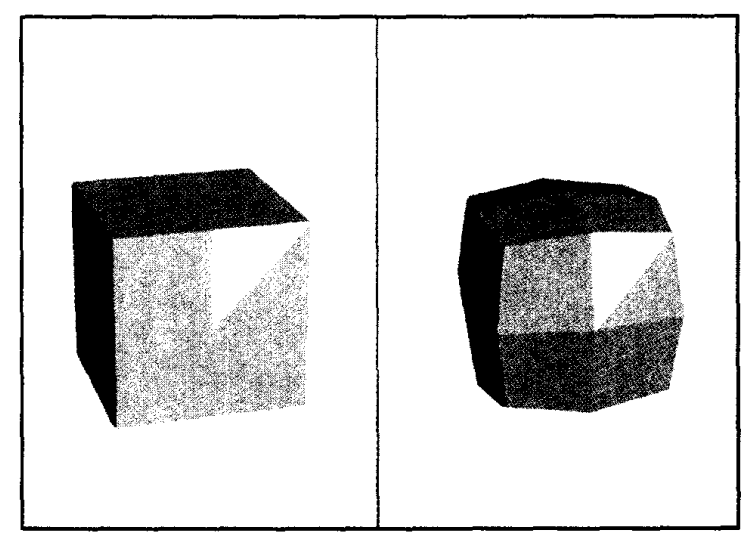

a

b
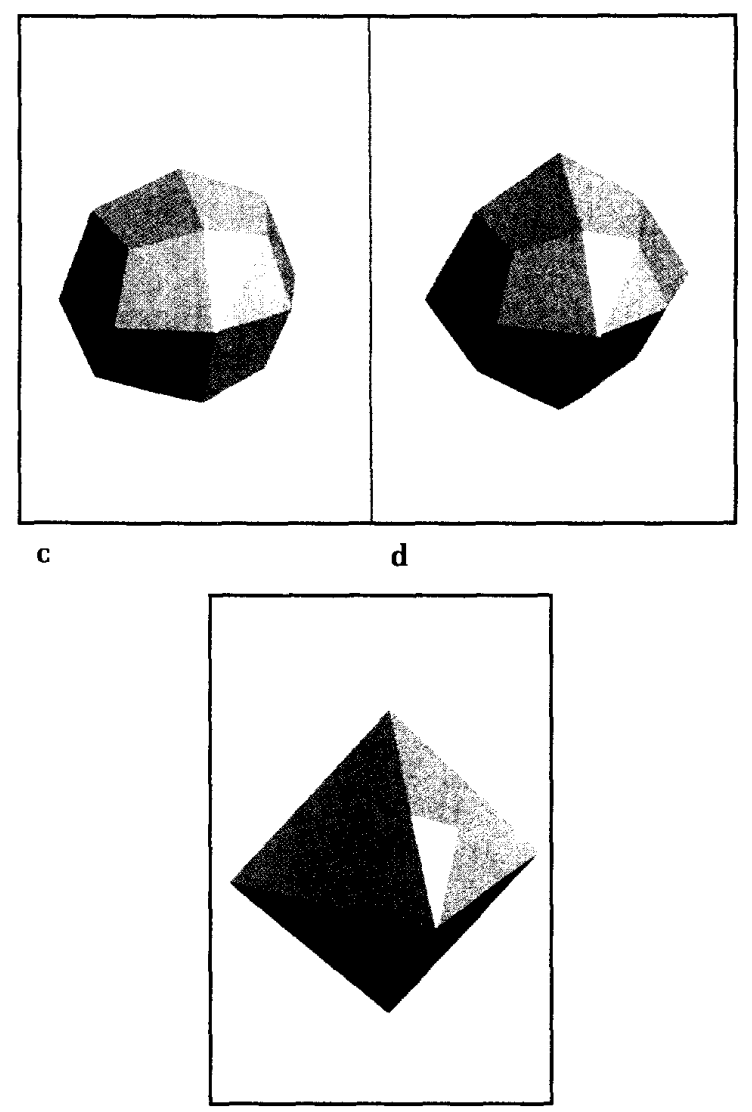

e

Figure 6.

triangle stays coplanar with the yellow one, each face of the cube breaks up into four quadrilaterals. The center point of each face bulges out, while the vertices flatten in, always keeping the midpoints of the edges of the cube unchanged. This sequence appears in Figure 6. A similar series can transform the octahedron to the rhombic dodecahedron and the rhombic dodecahedron to the cube. 


\section{Kaleidoscope}

Now let's go really wild! We can replace the seed triangle with any shape whatever, and all the replications will generate a result that also shares the symmetry of the cube. In Figure 7 I have tried a few variations on this theme. Figure $7 \mathrm{a}$ is just the cube recolored with normal triangles, in blue, and mirrored triangles in yellow. In Figure 7b I stuck in the bizarre values of $(a, b, c)=(-1.15,2,1.41)$. Figure $7 c$ relaxes the restrictions of triangle edges matching. Figure $7 d$ replaces the triangle with a sort of blunted pyramid shape. Much more fun could be had using teapots, but you get the idea.

Furthermore, by making the seed shape rotate and move about in some random way, you can animate a changing shape that generates the 3D kaleidoscope patterns of the title. I have seen several interesting test animations done this way, but the names of the perpetrators escape me.

\section{The tetrahedron}

I will briefly go through this rigamarole for another simple shape, the tetrahedron. A tetrahedron is exactly half as symmetrical as a cube. This is because only half of the 48 cube transformations will leave the tetrahedron intact (and in its original orientation). For the tetrahedron shown in Figure 8a they are as follows:

- The identity

- Rotations of $180^{\circ}$ about $x, y$ or z (3 total)

- Rotations of $\pm 120^{\circ}$ about the diagonals of an enclosing cube ( 8 total)

- Reflections about the $x= \pm y, x= \pm z$, and $y= \pm z$ planes (6 total)

- Reflections through the $x, y$, and $z$ axes followed by rotation of $\pm 90^{\circ}$ about that axis ( 6 total)

We can synthesize the tetrahedron and its symmetric relatives by starting with a seed triangle whose vertices are

Point 1: $(a, a, a)$

Point 2: $(0, b, 0)$

Point 3: $(c, c,-c)$

Combine this with its mirror image formed by reflecting across the $x=y$ plane. This generates one third of the equilateral triangle for one face:

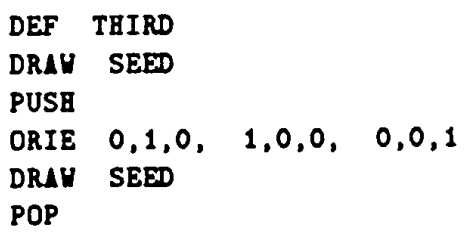

Generate the rest of the face by copies rotated $120^{\circ}$ about the center of the face, $(1 / 3,1 / 3,-1 / 3)$. The matrix to do this is surprisingly simple:

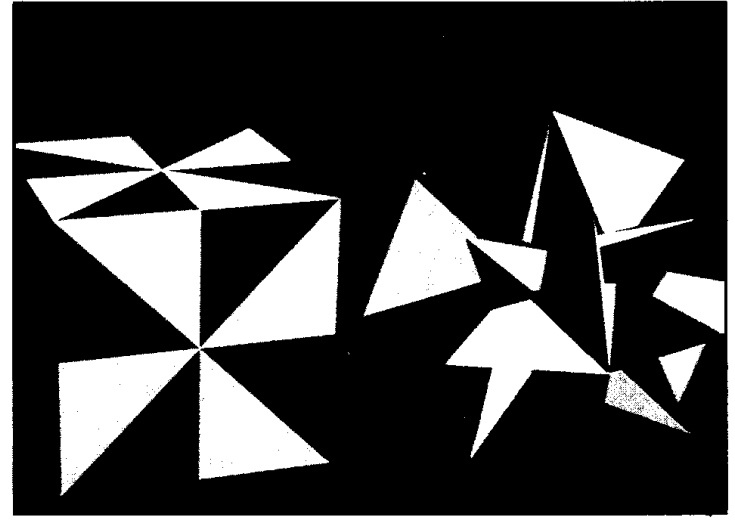

a $\quad$ b

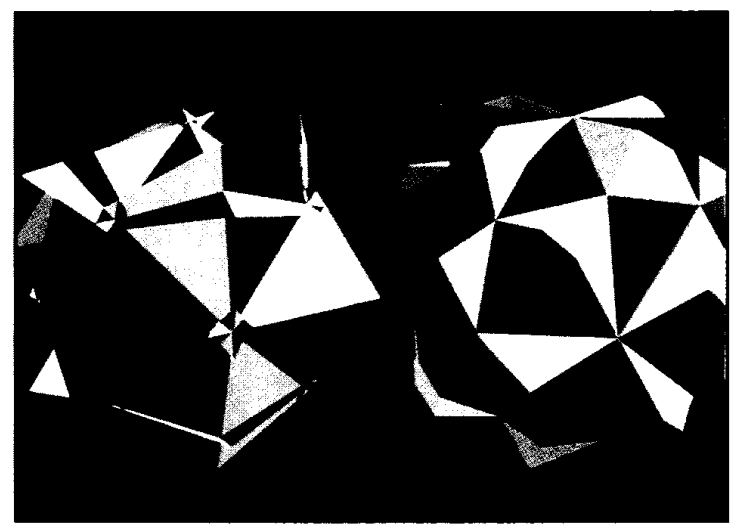

c

Figure 7.

$$
\left[\begin{array}{ccc}
0 & 1 & 0 \\
0 & 0 & -1 \\
-1 & 0 & 0
\end{array}\right]
$$

In transformation language:

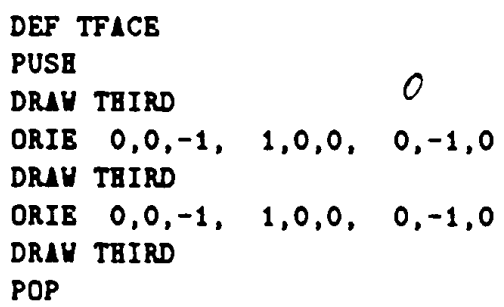

Finally copy this face four times to get the whole tetrahedron:

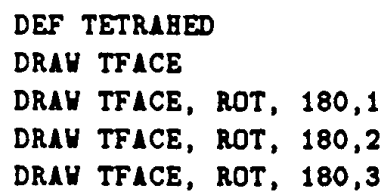




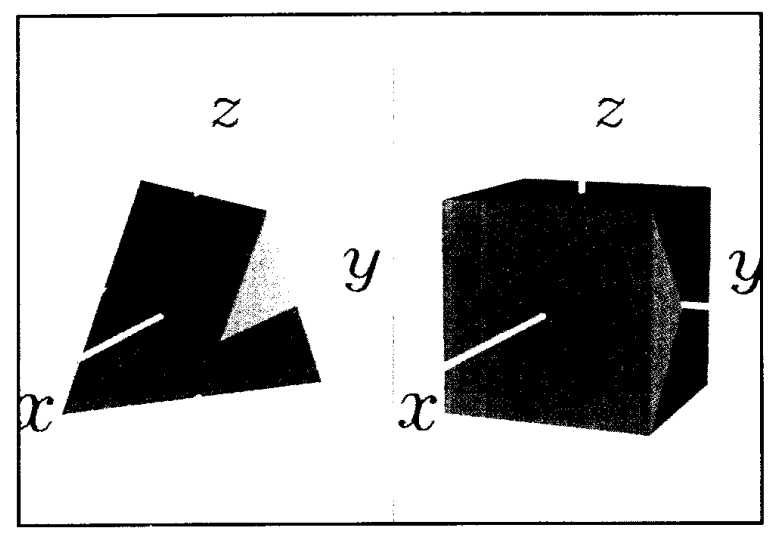

a

b

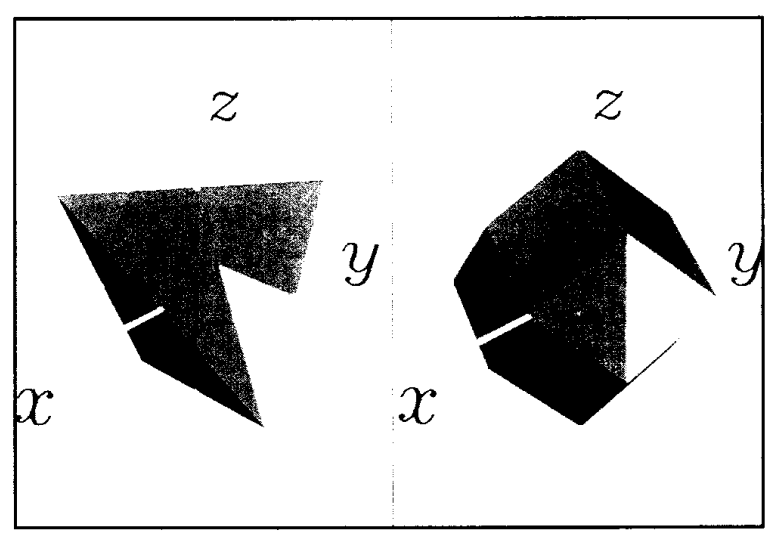

d
Again, by varying $a, b$ and $c$, you can get various shapes that share the symmetry of the tetrahedron. A coplanar-neighbor analysis done in a similar manner to the cube case gives three conditions as follows. First fix $b=1$. The conditions are

$$
\begin{aligned}
& \text { 1. } a=1 \\
& \text { 2. } c=1 \\
& \text { 3. } c=\frac{a}{4 a-1}
\end{aligned}
$$

If both conditions 1 and 3 are met, you get the tetrahedron. If both conditions 2 and 3 are met, you get another tetrahedron upside down (the dual of the original one). If both conditions 1 and 2 are met, you get a cube. You can also get a rhombic dodecahedron if $a=c=1 / 2$ (see Figure 8). Tabulating these conditions gives

$\begin{array}{ccccl}\text { condns } & a & b & c & \text { shape } \\ 1,3 & 1 & 1 & \frac{1}{3} & \text { Tetrahedron } \\ 2,3 & \frac{1}{3} & 1 & 1 & \text { Tetrahedron } \\ 1,2 & 1 & 1 & 1 & \text { Cube } \\ 3 & \frac{1}{2} & 1 & \frac{1}{2} & \text { Rhombic Dodecahedron }\end{array}$

Wait a minute...I just said that this group was only half as symmetrical as the cube. Now we have a cube coming out of it. What gives? Well, if $a=c$, the seed shape itself is now symmetrical (it has mirror symmetry along the $\mathrm{z}$ axis). I leave it to you to figure out if we could have done a similar thing with the cube symmetry above.

\section{Further ideas}

The examples shown here are just two of the many $3 \mathrm{D}$ point symmetry groups. The next logical candidate for exploration is the icosahedron-dodecahedron pair. What other shape does this generate in the same manner that the cube-octahedron pair generated the rhombic dodecahedron? What other non-Platonic shapes typify the other $3 \mathrm{D}$ point symmetry groups? If anybody out there generates some pictures of these, I will print them in a future column. 